\title{
AUTOMORPHISM GROUPS AND ANTI-PLURICANONICAL CURVES
}

\author{
DE-Qi ZHANG
}

\begin{abstract}
We show the existence of an anti-pluricanonical curve on every smooth projective rational surface $X$ which has an infinite group $G$ of automorphisms of either null entropy or of type $\mathbb{Z} \ltimes \mathbb{Z}$, provided that the pair $(X, G)$ is minimal. This was conjectured by Curtis T. McMullen (2005) and further traced back to Marat Gizatullin and Brian Harbourne (1987). We also prove (perhaps) the strongest form of the famous Tits alternative theorem.
\end{abstract}

\section{Introduction}

In this paper, we are interested in the automorphism groups of smooth projective complex surfaces. Especially, we are interested in the following question of GizatullinHarbourne-McMullen (see [16] page 409 and [24] §12), where $\operatorname{Aut}^{*}(X):=\operatorname{Im}(\operatorname{Aut}(X)$ $\rightarrow \operatorname{Aut}(\operatorname{Pic}(X)))$ :

Question 1.1. Let $X$ be a smooth projective rational surface. If $\operatorname{Aut}^{*}(X)$ is infinite, is there then a birational morphism $\varphi$ of $X$ to a surface $Y$ having an antipluricanonical curve and an infinite subgroup $G \subset \operatorname{Aut}^{*}(Y)$ such that $G$ lifts via $\varphi$ to $X ?$

A member in an anti-pluricanonical system $\left|-n K_{X}\right|(n \geq 1)$ is called an anti $n$-canonical curve (or divisor) or simply an anti-pluricanonical curve; a member in $\left|-K_{X}\right|$ is an anti 1-canonical curve, or an anti-canonical curve.

The result below answers Question 1.1 in the case of null entropy.

Theorem 1.2. Let $X$ be a smooth projective rational surface and $G \leq \operatorname{Aut}(X)$ an infinite subgroup of null entropy (see 2.5). Then we have:

(1) There is a $G$-equivariant smooth blowdown $X \rightarrow Y$ such that $K_{Y}^{2} \geq 0$ and hence $Y$ has an anti-pluricanonical curve.

(2) Suppose further that $\operatorname{Im}(G \rightarrow \operatorname{Aut}(\operatorname{Pic}(X)))$ is also an infinite group. Then the $Y$ in (1) can be so chosen that $-K_{Y}$ is nef of self intersection zero and $Y$ has an anti 1-canonical curve.

For groups which are not necessarily of null entropy, we have the following resul which is especially applicable (with the same kind of $H$ ) when $G / H \geq \mathbb{Z} \ltimes \mathbb{Z}$. See Theorems $5.3-5.5$ for more general results.

Received by the editors January 3, 2007.

1991 Mathematics Subject Classification. 14J26; 14J50, 37B40, 32H50.

Key words and phrases. automorphism, anti-pluricanonical curve, complex dynamics. 
Theorem 1.3. Let $X$ be a smooth projective surface and $G \leq \operatorname{Aut}(X)$ a subgroup. Assume that there is a sequence of groups

$$
H \unlhd A \unlhd G
$$

satisfying the following three conditions:

(1) $\operatorname{Im}(H \rightarrow \operatorname{Aut}(\mathrm{NS}(X)))$ is finite;

(2) $A / H$ is infinite and abelian; and

(3) $|G / A|=\infty$.

Then $G$ contains a subgroup $S$ of null entropy and infinite order.

In particular, when $X$ is rational, there is an $S$-equivariant smooth blowdown $X \rightarrow Y$ such that $Y$ has an anti-pluricanonical curve.

Remark 1.4. (1) Conditions like the ones in Theorems 1.2 and 1.3 are probably necessary in order to have an affirmative answer to Question 1.1. See Bedford-Kim [2, Theorem 3.2] for a pair $(X, g)$ with $g$ of positive entropy and Iitaka D-dimension $\kappa\left(X,-K_{X}\right)=-\infty$.

(2) The blowdown process $X \rightarrow Y$ to the minimal pair $(Y, S)$ in Theorems 1.2 and 1.3 is necessary, as observed by Harbourne [16].

However, the minimality assumption of the pair in Theorem 1.7 is not essential, though it makes the statement simpler.

To tackle Question 1.1 of Gizatullin-Harbourne-McMullen, we begin by determining the dynamical structure of surface automorphism groups. We first take a close look at McMullen's smooth rational surface $S^{v}$. It turns out that the dynamics of $\operatorname{Aut}\left(S^{v}\right)$ is very simple:

Theorem 1.5. Suppose that $v$ is a leading eigenvector for a Coxeter element $w$ in the Weyl group $W_{n}(n \geq 10)$.

Then for the McMullen surfaces $S^{v}$ of $[24, \S 7]$ (the surfaces $S_{n}$ in $[24$, Theorem 1.1] are among them), we have (semi-direct product):

$$
\operatorname{Aut}\left(S^{v}\right)=\left\langle h_{m}\right\rangle \ltimes T
$$

with $h_{m}$ of positive entropy and $T \triangleleft \operatorname{Aut}\left(S^{v}\right)$ a finite subgroup.

Our result below is (perhaps) the strongest form of the famous Tits [30] alternative theorem, for surface automorphisms groups of positive entropy. Let $\sigma: \operatorname{Aut}(X) \rightarrow$ Aut $\left(\mathrm{NS}_{\mathbb{Q}}(X)\right)$ be the natural homomorphism.

Note that the $T$ itself in (2) below is also finite when $X$ is a rational or $K 3$ surface (see Proposition 2.11; Sterk [29] Lemma 2.1, and Torelli theorem).

Theorem 1.6. Let $X$ be a smooth projective surface and $G \leq \operatorname{Aut}(X)$ a subgroup of positive entropy. Then $G$ satisfies either:

(1) $G$ contains the non-abelian free group $\mathbb{Z} * \mathbb{Z}$; or

(2) There is a $B \unlhd G$ such that $|G / B| \leq 2$ and $B=\left\langle h_{m}\right\rangle \ltimes T$ (semi-direct product) with $h_{m}$ positive entropy and $\sigma(T)$ finite. 
See Oguiso [27] Theorem 2.1 or 1.3 for groups of null entropy and Oguiso [26] Theorem 1.1 for $K 3$ groups (and more generally for hyperkähler manifolds), where the cyclic-ness of $B / T$ in our result here is replaced by abelian-ness. The Case(1) in the theorem above does occur (see Mazur [22], Cantat [6], Cantat - Favre [7] Example 3.2, and Oguiso [27] Theorem 1.6).

In Theorem 1.7 below we determine the relation between the anti- pluricanonical curve and the set $\operatorname{Stab}(g)$ of $g$-periodic curves.

Theorem 1.7. Let $X$ be a smooth projective rational surface with an automorphism $g$ of positive entropy. Assume the following two conditions:

(1) The pair $(X,\langle g\rangle)$ is minimal; and

(2) Either the set $\operatorname{Stab}(g)$ of $g$-periodic curves contains a curve of arithmetic genus $\geq 1$, or $X$ has an anti-pluricanonical curve.

Then we have:

(1) There is a unique nonzero effective $\mathbb{Q}$-divisor $\Delta$ with $\operatorname{Supp}(\Delta) \subseteq$ $\operatorname{Stab}(g)$ such that $K_{X}+\Delta \equiv 0$ (numerical equivalence).

(2) $\operatorname{Stab}(g)$ is a union of $\operatorname{Supp}(\Delta)$ and possibly a few $(-2)$-curves away from $\operatorname{Supp}(\Delta)$.

(3) Every anti-pluricanonical curve is a multiple of $\Delta$.

1.8. New development

In [25], [34], [35] and [20], we prove the conjecture of Amerik-Campana-FujimotoNakayama on the induced action on the base variety of an Iitaka fibration (generalizing the result of Deligne-Nakamura-Ueno), prove the conjecture of Guedj on cohomologically hyperbolic endomorphisms, and show some theorems of Tits type (generalizing results of Dinh-Sibony [11]).

\subsection{Acknowledgment}

I would like to thank Florin Ambro for patiently answering my questions on $\mathbb{R}$ divisors, Serge Cantat and JongHae Keum for constructive comments, Curtis T. McMullen for informing me about his new paper [24], Noboru Nakayama for answering my questions on strictly nef divisors, Keiji Oguiso for informing me of his result in [27] used in our Theorem 4.1, Helene Esnault and Eckart Viehweg for the support and warm hospitality during my stay in Essen, and the anonymous referee for kind suggestions.

The Main Theorem of [12] on automorphisms group $G$ (in the surface case, and with the assumption of the commutativity of $G$ ) agrees with the result of our Theorem 5.3 on $G$ (which is not necessarily commutative).

This project was supported by an Academic Research Fund of NUS.

\section{Conventions and Preliminary results}

2.1. Conventions are as in [17], [19] and [21].

Let $V$ be a normal projective variety. For an $\mathbb{R}$-Cartier divisor $D$ on $V, \operatorname{Null}(D):=$ $\{C \mid C$ is an irreducible curve with $C . D=0\}$ is the set of curves annihilated by $D$. Set $D^{\perp}:=\left\{E \in \mathrm{NS}_{\mathbb{Q}}(V) \mid E . D=0\right\}$. 
For an automorphism $g \in \operatorname{Aut}(V)$, a curve $C$ is $g$-periodic if $g^{s}(C)=C$ for some $s>0$. Denote by $\operatorname{Stab}(g):=\left\{C \subset V \mid C\right.$ is an irreducible curve with $g^{s}(C)=C$ for some $s>0\}$ the set of all $g$-periodic curves. For a divisor $M$ and subgroup $G \leq \operatorname{Aut}(V), \quad \operatorname{Stab}_{G}(M)=\left\{x \in G \mid x^{*} M \equiv \alpha_{x} M\right.$ in $\mathrm{NS}_{\mathbb{C}}(V)$ for some $\left.\alpha_{x} \in \mathbb{C}\right\}$ is the 'stabilizer' subgroup. For a group $G, Z(G):=\{g \in G \mid x g=g x$ for all $x \in G\}$ is the centre of $G$. If $H$ is a subgroup (resp. normal subgroup) of $G$, we denote $H \leq G$ (resp. $H \unlhd G$ ).

$\mathrm{A}(-n)$-curve $C$ on a surface is a curve with $C \cong \mathbb{P}^{1}$ and $C^{2}=-n$. A connected divisor on a surface is a rational tree, if it is of simple normal crossing, has the dual graph a tree and consists of smooth rational curves.

Definition 2.2. Let $X$ be a smooth projective surface and $G \leq \operatorname{Aut}(X)$ a subgroup. The pair $(X, G)$ is not minimal (resp. is minimal) if the equivalent conditions below are satisfied (resp. if neither of the conditions below is satisfied):

(1) There is a non-empty finite set $\Sigma$ of disjoint $(-1)$-curves on $X$ such that $\Sigma$ is $G$-stable, i.e., $G$ acts on $\Sigma$ as permutations.

(2) There is a $G$-equivariant non-isomorphic smooth blowdown $X \rightarrow Y$ onto a surface $Y$ endowed with a faithful $G$ action.

We use the format in KMM [19] Theorem 7-3-1 for Fujita's result in [14]:

Lemma 2.3. Let $X$ be a smooth projective surface and $D$ a pseudo effective $\mathbb{R}$-divisor. Then there is a unique effective $\mathbb{R}$-divisor $N$ satisfying:

(1) Either $N=0$, or the irreducible components $N_{i}$ of $N$ give rise to a negative definite intersection matrix $\left(N_{i} \cdot N_{j}\right)_{1 \leq i, j \leq s}$;

(2) $P:=D-N$ is nef; and

(3) $P . N=0$ (equivalently (using (2)), P. $N_{i}=0$ for all $i$ ).

Finally $N$ ard $P$ are $\mathbb{Q}$-Cartier divisors if so is $D$.

For $D$ in the lemma above, we write $D=P+N$ and call it the Zariski decomposition for pseudo effective divisor $D$. The uniqueness above also shows: if $D \equiv D^{\prime}$ with $D=P+N$ and $D^{\prime}=P^{\prime}+N^{\prime}$ their Zariski decompositions, then $N=N^{\prime}$ (equal) and $P \equiv P^{\prime}$.

For the result below, we may refer to BHPV [1], Ch IV, Cor. 7.2 and the CauchySchwartz inequality; see Kawaguchi [18] Lemma 1.2.

Lemma 2.4. Let $X$ be a smooth projective surface. Let $M$ and $D$ be $\mathbb{R}$-divisors on $X$ neither of which is numerically trivial. Suppose that $M$ is nef, $D$ is pseudo-effective and $M . D=0$. The we have:

(1) $D^{2} \leq 0$; and

(2) $D^{2}=0$ holds if and only if $M^{2}=0$ and $D \equiv a M$ for some $a>0$.

Definition 2.5. Topological entropy. Let $M$ be a compact Kähler manifold. Let $\lambda(g)$ be the spectral radius of the action $g^{*}$ on the cohomology ring $H^{*}(M, \mathbb{C})$, i.e., the maximum of moduli of its eigenvalues. The topological entropy of $g$ is defined as $e(g)=\log \lambda(g)$. See [15], [31] [13], also [12]. 
It is known that $e(g) \geq 0$, and $e(g)=0$ if and only if every eigenvalue of the action $g^{*}$ above has modulus 1 . Also $e(g)>0$ if and only if at least one eigenvalue of the restriction $g^{*} \mid H^{1,1}(M)$ has modulus different from 1; see Dinh and Sibony [11].

An element $g$ is of null (resp. positive) entropy if $e(g)=0$ (resp. $e(g)>0$ ). A subgroup $G \leq \operatorname{Aut}(M)$ is of null entropy (resp. positive entropy) if $e(g)=0$ for all $g \in G$ (resp. $e(g)>0$ for at least one $g \in G$ ).

When $\bar{M}$ is a normal projective surface and $g \in G \leq \operatorname{Aut}(\bar{M})$, we say $g$ (resp. $G$ ) is of null or positive entropy if so is $g$ (resp. $G$ ) regarded as an element (resp. a subgroup) of Aut $(M)$. Here $M \rightarrow \bar{M}$ is Hironaka's equivariant resolution and induces the natural inclusion $\operatorname{Aut}(\bar{M}) \subseteq \operatorname{Aut}(M)$.

2.6. Assumption. From now on till Proposition 2.11 (but except Lemma 2.8), we assume that $X$ is a smooth projective surface with an (infinite) automorphism $g$ such that $g^{*} \mid H^{1,1}(X)$ has an eigenvalue $\lambda$ with $|\lambda|>1$. Namely, assume that $g$ is of positive entropy. In Lemma 2.7 below, it turns out that such $\lambda$ with modulus $>1$ is unique, and we can write $\lambda=\lambda(g)$.

When $X$ is only a normal projective surface with $\tilde{X} \rightarrow X$ the minimal resolution, we let $\lambda(g)=\lambda\left(g_{\tilde{X}}\right)$; here $g \in \operatorname{Aut}(X)$ induces $g_{\tilde{X}} \in \operatorname{Aut}(\tilde{X})$.

We remark that this $\lambda$ is an algebraic number and is either a Pistol number or a Salem number according to the degree of $\lambda$ over $\mathbb{Q}$ (degree 2 or bigger). See Salem [28] and McMullen [24] $§ 2$.

For the proof of the result below and the current formulation of it, see Cantat [5] Theorem 2.1.5, Dinh and Sibony [11] Theorem 2.1 and McMullen [23] Theorem 3.2 and Corolalry 3.3, or Kawaguchi [18] Theorem 2.1.

Lemma 2.7. Let $X, g$ be as in 2.6. Then $\lambda=\lambda(g)>1 ; \lambda$ and $\lambda^{-1}$ are conjugate over $\mathbb{Q}$. Further, setting $h=h^{1,1}(X)$, the following are all eigenvalues of $g^{*} \mid H^{1,1}(X)$ (with each $\left|\alpha_{j}\right|=1$; in particular, $\left.\lambda\left(g^{-1}\right)=\lambda(g)\right)$ :

$$
\lambda, \lambda^{-1}, \alpha_{1}, \alpha_{2}, \ldots, \alpha_{h-2}
$$

For the proof of the result below, see Cantat [4] Theorem 2, Diller and Favre [9] Theorem 5.1, or Kawaguchi [18] Proposition 2.5 and Lemma 3.8.

Lemma 2.8. Let $X$ be a normal projective surface and $g$ an automorphism with positive entropy $\log (\lambda)$. Then we have:

(1) There exist nef $\mathbb{R}$-Cartier divisors $L^{+}=L(g)^{+}$and $L^{-}=L(g)^{-}$(unique upto to positive scalars), which are not numerically trivial, such that the following are true (especially, $L\left(g^{ \pm}\right)^{+}=L\left(g^{\mp}\right)^{-}$):

$$
g^{*} L^{+} \equiv \lambda L^{+}, \quad g^{*} L^{-} \equiv \lambda^{-1} L^{-} .
$$

(2) Let $\sigma: \tilde{X} \rightarrow X$ be the minimal resolution so that $g \in \operatorname{Aut}(X)$ induces $g_{\tilde{X}} \in$ $\operatorname{Aut}(\tilde{X})$. Then $L\left(g_{\tilde{X}}\right)^{ \pm}$equals $\sigma^{*} L(g)^{ \pm}$up to positive scalars.

Indeed, by the proof in Kawaguchi, Lemma 2.4 and reducing to $\tilde{X}$ (see also Cantat [4] Theorem 2 and Diller and Favre [9] Theorem 5.1), we can start with any ample 
(or even big $\mathbb{R}-$ ) Cartier divisor $B$ on $X$ and take $L^{+}$and $L^{-}$as follows:

$$
L^{+}=\lim _{n \rightarrow+\infty} \frac{\left(g^{*}\right)^{n}(B)}{\lambda^{n}}, L^{-}=\lim _{n \rightarrow+\infty} \frac{\left(g^{*}\right)^{-n}(B)}{\lambda^{n}} .
$$

By the description above, we have the following (see Kawaguchi [18] Proposition 2.5; use also the Hodge index theorem for (3) - (4)):

Lemma 2.9. Let $X, g$ be as in 2.6. The following are true.

(1) $\left(L^{+}\right)^{2}=0=\left(L^{-}\right)^{2}$.

(2) $L:=L^{+}+L^{-}$is nef and big (i.e., $L^{2}>0$ ).

(3) Suppose that $D$ is an $\mathbb{R}$-divisor on $X$ such that $\left(g^{s}\right)^{*} D \equiv D$ for some $s>0$.

Then $L^{+} . D=0=L^{-} . D$, so either $D^{2}<0$ or $D \equiv 0$.

(4) $L^{+} . K_{X}=0=L^{-} . K_{X}$; so either $K_{X}^{2}<0$ or $K_{X} \equiv 0$.

Here is the relation between $\operatorname{Stab}(g)$ and $\operatorname{Null}\left(L^{ \pm}\right)$:

Lemma 2.10. Let $X, g$ be as in 2.6. The following are true.

(1) $\operatorname{Stab}(g)=\operatorname{Null}\left(L^{+}\right) \cap \operatorname{Null}\left(L^{-}\right)=\operatorname{Null}(L)$, where $L=L^{+}+L^{-}$.

(2) $\operatorname{Null}(L)$ is either empty, or a finite set with negative intersection matrix. In particular, $|\operatorname{Null}(L)|<\rho(X)$, the Picard number.

(3) If $\operatorname{Null}(M)$ is a finite set for $M=L^{+}$or $M=L^{-}$(this is always true by Theorem 6.2), then $\operatorname{Null}(M)=\operatorname{Stab}(g)$.

(4) The pair $(X,\langle g\rangle)$ is minimal if and only if $\operatorname{Stab}(g)$ does not contain any $(-1)$ curve.

Proof. (2) is the consequence of the Hodge index theorem and that $L^{2}>0$. The nefness of $L^{ \pm}$implies the second equality in (1). Clearly, $g$ stabilizes each of the sets $\operatorname{Null}\left(L^{+}\right), \operatorname{Null}\left(L^{-}\right)$and $\operatorname{Null}(L)$. So (1) and (3) follow (see (2) and Lemma 2.9; see Kawaguchi [18] Proposition 3.1). The set $\Sigma$ (possibly empty) consisting of all $(-1)$-curves in $\operatorname{Stab}(g)$, is $g$-stable. So (4) is just the definition in 2.2.

We prove several frequently-used properties of $g$ of positive entropy. The assertion (4) below follows from the proof of Kawaguchi [18] Claim 3.8.1. Denote by $P_{g}(x)$ the characteristic polynomial of $g^{*} \mid \mathrm{NS}_{\mathbb{Q}}(X)$, and $f_{g}(x)$ the minimal (irreducible) polynomial of $\lambda(g)$ over $\mathbb{Q}$.

Proposition 2.11. Let $X, g, \lambda, L^{ \pm}$be as in 2.6. The following are true.

(1) For $r \geq 1$ one has $P_{g^{r}}(x)=f_{g^{r}}(x) q_{g^{r}}(x)$ where $q_{g^{r}}(x)$ is a product of cyclotomic polynomials while $f_{g^{r}}(x)$ has no root of 1 . One has $q_{g^{s}}(x)=(x-1)^{e}$ for some positive integers $s=s(g)$ and $e=e(g)$. Also $\operatorname{deg}\left(q_{g^{r}}(x)\right)=e$ for all $r \geq 1$.

(2) Further, we have the decomposition into two g-stable $\mathbb{Q}$-spaces

$$
\mathrm{NS}_{\mathbb{Q}}(X)=V_{g} \oplus V_{g^{s}=1}
$$

such that $\left(g^{r}\right)^{*} \mid V_{g}(r \geq 1)$ has $f_{g^{r}}(x)$ as its characteristic polynomial and also minimal polynomial, the inclusion $\left(^{*}\right): V_{g} \otimes_{\mathbb{Q}} \mathbb{R} \supset \mathbb{R}\left[L^{+}\right] \oplus \mathbb{R}\left[L^{-}\right]$, $e=\operatorname{rank}_{\mathbb{Q}} V_{g^{s}=1}$, and

$$
V_{g^{s}=1}=\left\{v \in \mathrm{NS}_{\mathbb{Q}}(X) \mid g^{s}(v)=v\right\}=\left(L^{+}\right)^{\perp} \cap\left(L^{-}\right)^{\perp} \cap \mathrm{NS}_{\mathbb{Q}}(X) .
$$


(3) If $X$ is rational then $\operatorname{Ker}(\operatorname{Aut}(X) \rightarrow \operatorname{Aut}(\operatorname{Pic}(X)))$ is finite.

(4) We may take $L^{ \pm}$to be in $(\operatorname{Pic}(X)) \otimes_{\mathbb{Z}} \mathbb{Z}[\lambda]$.

(5) $\lambda$ is not a rational number.

(6) None of positive multiples of $L^{ \pm}$is $\mathbb{Q}$-Cartier.

Proof. (1) The first part follows from Lemma 2.7 and Kronecker's theorem; see McMullen [23] Corollary 3.3. For the second, let $s$ be the $1 \mathrm{~cm}$ of orders of the roots in $q_{g}(x)$.

(2) By (1), we have $\mathrm{NS}_{\mathbb{Q}}(X)=V_{r}^{\prime} \oplus V_{r}^{\prime \prime}$ (each summand being $g$-stable) so that $f_{g^{r}}(x)$ and $q_{g^{r}}(x)$ are respectively the characteristic polynomials of $\left(g^{r}\right)^{*} \mid V_{r}^{\prime}$ and $\left(g^{r}\right)^{*} \mid V_{r}^{\prime \prime}$. Further, we have $V_{i}^{\prime}=V_{j}^{\prime}$ (denoted as $V_{g}$ ) and $V_{i}^{\prime \prime}=V_{j}^{\prime \prime}$ (denoted as $V_{g^{s}=1}$ ) for all $i, j \geq 1$, since the only $g$-stable vector subspaces of $V_{r}^{\prime}$ are $\{0\}$ and itself by the irreducibility of $f_{g^{r}}(x)$.

Since the classes $\left[L^{ \pm}\right]$are eigenvectors w.r.t. eigenvalues $\lambda^{ \pm}$of $g^{*}$, we have the inclusion $\left(^{*}\right)$ in $(2)$.

For the second equality in the second display of (2), in view of Lemma 2.9, we have only to show the assertion that $\left(g^{r}\right)^{*} \mid W=$ id for some $r \geq 1$, where $W:=$ $\left(L^{+}\right)^{\perp} \cap\left(L^{-}\right)^{\perp} \cap \mathrm{NS}(X)$. By the Hodge index theorem, $W$ (modulo its finite torsion) is a negative definite integral lattice. So $\operatorname{Aut}(W)$ is finite and the assertion is true. Thus the second equality is proved.

Now the first equality (even when $s$ is replaced by $s n$ with $n \geq 1$ ) follows from the second, the choice of $s$ and the application of Lemma 2.9 (3) inductively on the size of the Jordan canonical form of $g^{*} \mid V_{r}^{\prime \prime} \otimes_{\mathbb{Q}} \mathbb{C}$.

(3) Suppose the contrary that this Kernel is infinite. Then $X$ has finitely many 'exceptional curves' by Harbourne [16] Proposition 1.3 and its terminology at the last paragraph of page 409. Since $g$ acts on the finite set of these curves, for some common $m \geq 1$, each irreducible component of these exceptional curves is stabilized by $g^{m}$. Let $X \rightarrow Y$ be the $\left\langle g^{m}\right\rangle$-equivariant smooth blowdown to a relatively minimal model. Then $K_{Y}^{2} \geq 8$, while $K_{Y}^{2}<0$ by Lemma 2.9 for $g^{m}$ being of positive entropy on $Y$. It is absurd.

(5) If $\lambda \in \mathbb{Q}$, then $\lambda \in \mathbb{Z}$ because it is algebraic over $\mathbb{Q}$, so $x-\lambda$ is its minimal polynomial over $\mathbb{Q}$, contradicting the fact that $\lambda$ and $\lambda^{-1}$ are conjugate over $\mathbb{Q}$ in Lemma 2.7 .

(6) If $L^{+}$is $\mathbb{Q}$-Cartier say, then by intersecting $g^{*} L^{+} \equiv \lambda L^{+}$with a Cartier ample divisor $H$, we see that $\lambda=\left(H . g^{*} L^{+}\right) /\left(H . L^{+}\right)$is a rational number. This contradicts (5). The proposition is proved.

Here is another consequence of the uniqueness result of Lemma 2.8 .

Lemma 2.12. Let $X$ be a smooth projective surface, $M$ a nef $\mathbb{R}$-divisor which is not numerically trivial, and $g \in \operatorname{Aut}(X)$ such that $g^{*} M \equiv \alpha M$ for some $\alpha \in \mathbb{C}$. Then we have:

(1) Either $\alpha=1$, or $\alpha>1$, or $0<\alpha<1$.

(2) If $\alpha=1$, then $g$ is of null entropy.

(3) If $\alpha^{ \pm}>1$, then $g$ is of positive entropy with $\lambda(g)=\alpha^{ \pm}$and $M$ is equal to $L(g)^{ \pm}$(up to a positive scalar). 
Proof. Intersecting the equality $g^{*} M \equiv \alpha M$ with an ample divisor $H$, we get $\alpha=$ $\left(H . g^{*} M\right) /(H . M)>0$. Then the lemma follows from Lemmas $2.7-2.9$ and that $M^{2} \geq 0$ for $M$ being nef.

The result below shows that when dealing with automorphisms of positive entropy we may quotient away a finite group.

Let $\sigma: \operatorname{Aut}(X) \rightarrow \operatorname{Aut}(\mathrm{NS}(X))$ be the natural homomorphism. For $g \in G \leq$ $\operatorname{Aut}(X)$, set $\tilde{g}=\sigma(g)$ and $\tilde{G}=\sigma(G)$.

Lemma 2.13. Let $X$ be a smooth projective surface and $H \leq \operatorname{Aut}(X)$ a subgroup such that $\sigma(H)=\tilde{H}$ is a finite subgroup of $\operatorname{Aut}(\operatorname{NS}(X))$. Suppose that $\sigma(g)=\tilde{g} \in$ $N_{\text {Aut }(\mathrm{NS}(X))}(\tilde{H})$ (i.e., $\left.\tilde{g} \tilde{H} \tilde{g}^{-1}=\tilde{H}\right)$. Then we have:

(1) If $g$ is of positive entropy, then for both $M=L(g)^{+}$and $M=L(g)^{-}$we have $h^{*} M \equiv M$ for all $h \in H$.

(2) Suppose that $H$ is already finite. Let $\pi: X \rightarrow \bar{X}=X / H$ be the quotient map. Then $g$ is of positive entropy if and only if so is $\bar{g}=g H \in \bar{G}=$ $G / H \leq \operatorname{Aut}(\bar{X})$. If this is the case, we have $\lambda(g)=\lambda(\bar{g})$ and can take $L(g)^{ \pm}=\pi^{*} L(\bar{g})^{ \pm}$.

Proof. (1) Consider the case $M=L(g)^{+}$(the other case is similar), so $g^{*} M \equiv$ $\lambda M$ with $\lambda=\lambda(g)>1$. Set $M^{\prime}=\sum_{x \in \tilde{H}} x^{*}(M)$ (identifying $M$ with its class in $\mathrm{NS}(X) \otimes_{\mathbb{Z}} \mathbb{R}$ ). Then we have (where $h \in H$ ):

$$
\begin{gathered}
g^{*}\left(M^{\prime}\right)=\sum_{x \in \tilde{H}}\left(\tilde{g}^{-1} x \tilde{g}\right)^{*} \tilde{g}^{*} M \equiv \lambda \sum_{y \in \tilde{H}} y^{*} M=\lambda M^{\prime}, \\
h^{*}\left(M^{\prime}\right)=\sum_{x \in \tilde{H}}(x \tilde{h})^{*} M=\sum_{y \in \tilde{H}} y^{*} M=M^{\prime} .
\end{gathered}
$$

Clearly, $M^{\prime}$ is nef and is not numerically trivial, so the uniqueness Lemma 2.8 implies that $M \equiv \alpha M^{\prime}$ for some $\alpha>0$. Thus (1) follows.

(2) Suppose that $\bar{g}$ is of positive entropy. So $\bar{g}^{*} L(\bar{g})^{+} \equiv \lambda L(\bar{g})^{+}$with $\lambda=\lambda(\bar{g})>1$. Set $L(g)^{+}=\pi^{*} L(\bar{g})^{+}$which is nef and is not numerically trivial. Then $g^{*} L(g)^{+} \equiv$ $\lambda L(g)^{+}$because $\pi \circ g=\bar{g} \circ \pi$. Thus $g$ is of positive entropy $\log \lambda(g)=\log \lambda$.

Conversely, suppose that $g$ is of positive entropy $\log \lambda=\log \lambda(g)$. We shall use the fact below about the $H$-invariant sublattice

$$
\left((\operatorname{Pic}(X))^{H} \otimes_{\mathbb{Z}} \mathbb{R}=\pi^{*}(\operatorname{Pic}(\bar{X})) \otimes_{\mathbb{Z}} \mathbb{R} .\right.
$$

By the proof of (1), $L(g)^{+}$(re-chosen like $M^{\prime}$ above) belongs to the LHS above, so it is also in the RHS. Thus $L(g)^{+}=\pi^{*} \bar{L}$ for some $\mathbb{R}$-Cartier divisor $\bar{L}$. Since $\pi \circ g=\bar{g} \circ \pi$, we have $\pi^{*}(\lambda \bar{L}) \equiv \pi^{*}\left(\bar{g}^{*} \bar{L}\right)$. Hence $\bar{g}^{*} \bar{L} \equiv \lambda \bar{L}$ by the injectivity of $\pi^{*}:(\operatorname{Pic}(\bar{X})) \otimes_{\mathbb{Z}} \mathbb{R} \rightarrow(\operatorname{Pic}(X)) \otimes_{\mathbb{Z}} \mathbb{R}$. So $\bar{g}$ is of positive entropy. Thus $(2)$ and the lemma are proved, since the case $L(g)^{-}$is similar.

\section{Groups stabilizing nef classes; proof of Theorem $\mathbf{1 . 5}$}

In this section, we will determine the dynamical structure of the stabilizer subgroup $\operatorname{Stab}_{G}(M)$ when $M$ is nef and is not numerically trivial.

We will also determine the dynamical group structure of the full automorphism group $\operatorname{Aut}\left(S^{v}\right)$ for McMullen's surfaces $S^{v}$ in [24] $\S 7$. 
The following result is very important in proving our main theorems.

Theorem 3.1. Let $X$ be a smooth projective surface and $G \leq \operatorname{Aut}(X)$ a subgroup. Let $M$ be a nef $\mathbb{R}$-divisor which is not numerically trivial. Suppose that $G=\operatorname{Stab}_{G}(M)$ and $G$ is of positive entropy. Then we have:

(1) One has $G=\left\langle h_{m}\right\rangle \ltimes T$ (semi-direct product) with $h_{m}$ of positive entropy (and $\left.M=L\left(h_{m}\right)^{+}\right)$and $\operatorname{Im}(T \rightarrow \operatorname{Aut}(\mathrm{NS}(X)))$ finite.

(2) For $g \in G$, one has $g \in T$ if and only if $g$ is of null entropy; if and only if $g^{*} M \equiv M$ holds.

(3) Set $h:=h_{m}$. Then $T \mid V_{h}=\mathrm{id}$. Here $\mathrm{NS}_{\mathbb{Q}}(X)=V_{h} \oplus V_{h^{s(h)}=1}$ is as in 2.11 .

Proof. By the assumption, $G$ contains an automorphism $g$ of positive entropy $\log \lambda(g)$ $>0$. By Lemma 2.12 and switching $g$ with $g^{-1}$ if necessary, we may assume that $M=L(g)^{+}$and $g^{*} M \equiv \lambda(g) M$. Also, in the definition of $\operatorname{Stab}_{G}(M)$, either $\alpha_{x}=1$ and $x$ is of null entropy, or $\alpha_{x}^{ \pm}>1$ and $x$ is of positive entropy $\lambda(x)=\alpha_{x}^{ \pm}$.

Consider the map $\varphi: G \rightarrow \mathbb{R}$ which takes $x$ to $\log \alpha_{x}$ if $x^{*} M \equiv \alpha_{x} M$. Clearly, $\varphi$ is a homomorphism onto an abelian subgroup of the (torsion-free) additive group $\mathbb{R}$.

We assert that $\operatorname{Im}(\varphi)$ does not contain a subgroup of the type $\mathbb{Z} \times \mathbb{Z}$. Suppose the contrary that $\left\langle\log \left(\alpha_{g_{1}}\right)\right\rangle \times\left\langle\log \left(\alpha_{g_{2}}\right)\right\rangle \cong \mathbb{Z} \times \mathbb{Z}$ is a subgroup of $\operatorname{Im}(\varphi)$. Here $g_{i} \in G$ with $g_{i}^{*} M \equiv \alpha_{g_{i}} M$. Thus $1, \log \left(\alpha_{g_{2}}\right) / \log \left(\alpha_{g_{1}}\right)$ are linearly independent over $\mathbb{Q}$. Now by the classical Kronecker's Theorem (or Dirichlet's Theorem), for any $\varepsilon>0$, there are integers $m_{i}$ such that:

$$
\left|m_{2} \frac{\log \left(\alpha_{g_{2}}\right)}{\log \left(\alpha_{g_{1}}\right)}-m_{1}\right|<\varepsilon .
$$

After relabelling, we may assume that:

$$
0<m_{1} \log \left(\alpha_{g_{1}}\right)-m_{2} \log \left(\alpha_{g_{2}}\right)<\varepsilon_{1}:=\varepsilon \max \left\{\left|\log \left(\alpha_{g_{1}}\right)\right|,\left|\log \left(\alpha_{g_{2}}\right)\right|\right\} .
$$

Thus for $h=g_{1}^{m_{1}} / g_{2}^{m_{2}} \in G$, we have $h^{*} M \equiv \alpha_{h} M$ where $\alpha_{h}:=\alpha_{g_{1}}^{m_{1}} / \alpha_{g_{2}}^{m_{2}}$ satisfies $1<\alpha_{h}<e^{\varepsilon_{1}}$. So $h$ is of positive entropy with $\lambda(h)=\alpha_{h}$. On the other hand, in [24] Theorem 1.2 , McMullen has proved that $\lambda(f) \geq \lambda$ (Lehmer) $\approx 1.17628081$ for every surface automorphism $f$ of positive entropy. We get a contradiction if we let $\varepsilon$ (and hence $\left.\varepsilon_{1}\right)$ tend to zero. This proves the assertion.

Since $\varphi$ factors through $G \subseteq \operatorname{Aut}(X) \rightarrow \operatorname{Aut}(\mathrm{NS}(X))$ while the latter is countable, we can write $\operatorname{Im}(\varphi)=\cup_{n \geq 1} \bar{G}_{n}$ where each $\bar{G}_{n} \leq \mathbb{R}$ is finitely generated (and abelian). By the assertion above and noting that $\varphi(g)=\log \lambda(g)>0$ for some $g \in G$ (and the fundamental theorem for f.g. abelian groups) we may assume that $\bar{G}_{n}=\left\langle\log \lambda\left(g_{n}\right)\right\rangle$ for some $g_{n} \in G$ of positive entropy $\log \lambda\left(g_{n}\right)$. Now $\bar{G}_{n} \leq \bar{G}_{n+1}$ implies, by induction, that $\lambda\left(g_{n}\right)=\left(\lambda\left(g_{1}\right)\right)^{1 / s_{n}}$ for some positive integer $s_{n}$. Applying McMullen's result above again, we have $\left(\lambda\left(g_{1}\right)\right)^{1 / s_{n}}=\lambda\left(g_{n}\right) \geq \lambda$ (Lehmer) $>1.1$ for all $n \geq 1$. Thus there is a constant $N$ such that $s_{N}=s_{N+1}=\ldots$, whence $\operatorname{Im}(\varphi)=\bar{G}_{N}=\left\langle\log \lambda\left(h_{m}\right)\right\rangle$, where we set $h_{m}:=g_{N}$ and may assume that $M=L\left(h_{m}\right)^{+}$.

Set $T:=\operatorname{Ker}(\varphi)$. Then $T$ is of null entropy; so the assertion (2) follows (see also Lemma 2.12). Since $h_{m}$ is of positive entropy, $\left\langle h_{m}\right\rangle \cap T=(1)$ and hence $G=\left\langle h_{m}\right\rangle \ltimes T$. This proves (1) (except the finiteness of $T \mid \mathrm{NS}(X)$ ).

(3) Write $h:=h_{m}$ for simplicity. We use the notation in Proposition 2.11. Note that $f(x):=f_{h}(x)$ is the minimal polynomial of $\lambda=\lambda(h)$ over $\mathbb{Q}$ (hence $f(x)$ has only simple zeros by the Galois theory) and also the minimal polynomial of $h^{*} \mid V_{h}$, whence 
$V_{h} \otimes_{\mathbb{Z}} K(f)$ is spanned by the eigenvectors $v_{\beta}$ w.r.t. eigenvalues $\beta$ for $h^{*} \mid V_{h} \otimes_{\mathbb{Z}} K(f)$. Here $K(f)$ is the splitting field $(\subset \mathbb{C})$ of $f(x)$ over $\mathbb{Q}$.

So we have only to show the assertion that $t^{*} v_{\beta} \equiv v_{\beta}$ for all such eigenvector $v_{\beta}$ (so that $n t^{*} v_{\beta}$ and $n v_{\beta}$ are algebraically equivalent for some $n>0$ and hence $t^{*} v_{\beta}$ equals $v_{\beta}$ in $\left.\mathrm{NS}_{\mathbb{Q}}(X)\right)$. There is a $\gamma=\gamma_{h}$ in the Galois group $\operatorname{Gal}(f)=\operatorname{Gal}(K(f) / \mathbb{Q})$ of $f(x)$ such that $\gamma^{-1}(\beta)$ equals the spectral radius $\lambda$ (see Lemma 2.7). Set $L^{ \pm}=L(h)^{ \pm} \in$ $(\operatorname{Pic}(X)) \otimes_{\mathbb{Z}} \mathbb{Z}[\lambda]$ (see Proposition 2.11), so that $h^{*} L^{ \pm} \equiv \lambda^{ \pm} L^{ \pm}$. For $g \in \operatorname{Aut}(X)$, extend $g^{*} \mid \operatorname{Pic}(X) \quad \mathbb{C}$-linearly to $g^{*} \mid(\operatorname{Pic}(X)) \otimes_{\mathbb{Z}} \mathbb{C}$. For a $\mathbb{C}$-divisor $D=\sum c_{i} D_{i}$ with $D_{i} \in \operatorname{Pic}(X)$ and $c_{i} \in K(f)$, define $\gamma^{*}(D)=\sum \gamma\left(c_{i}\right) D_{i}$. Then $\gamma \circ g=g \circ \gamma$. Now:

$$
h^{*} \gamma^{*}\left(L^{+}\right)=\gamma^{*} h^{*}\left(L^{+}\right) \equiv \gamma^{*}\left(\lambda L^{+}\right)=\beta \gamma^{*}\left(L^{+}\right) .
$$

So $v_{\beta}:=\gamma^{*}\left(L^{+}\right)$(its class, to be precise) is an eigenvector w.r.t. $\beta$ for $h^{*} \mid \operatorname{NS}(X) \otimes_{\mathbb{Z}}$ $K(f)$. For $t \in T$, we have $t^{*} v_{\beta}=t^{*} \gamma^{*}\left(L^{+}\right)=\gamma^{*} t^{*}\left(L^{+}\right) \equiv \gamma^{*}\left(L^{+}\right)=v_{\beta}$. The assertion and hence $(3)$ are proved.

Finally, to show $T \mid \mathrm{NS}(X)$ is finite it is enough to show that $T \mid \mathrm{NS}_{\mathbb{Q}}(X)$ is finite, since $\mathrm{NS}(X)$ has a finite torsion. By (3) and the proof of Proposition 2.11, we then have only to show $T$ stabilizes $W:=\left(L^{+}\right)^{\perp} \cap\left(L^{-}\right)^{\perp} \cap \mathrm{NS}(X)$ (noting that $W \otimes_{\mathbb{Z}} \mathbb{Q}=V_{h^{s(h)}}=1$ and $O(W)$ is finite). This is clear because $t^{*} L^{ \pm} \equiv L^{ \pm}$for $t \in T$ by (3) and Proposition 2.11 (2). We are done.

By the proof above, $\left\langle h_{m}\right\rangle \ltimes T$ stabilizes (up to scalars) $L\left(h_{m}\right)^{-}$. Reversing the process or noting that $L\left(h_{m}\right)^{-}=L\left(h_{m}^{-1}\right)^{+}$, one obtains:

Corollary 3.2. Let $X$ be a smooth projective surface and $g$ an automorphism of positive entropy. Then $\operatorname{Stab}_{\text {Aut }(X)}\left(L(g)^{+}\right)=\operatorname{Stab}_{\text {Aut }(X)}\left(L(g)^{-}\right)$.

We now elaborate further about the stabilizer subgroup $\operatorname{Stab}_{G}(M)$.

Proposition 3.3. Assume that $G=\operatorname{Stab}_{G}(M)$ and $G$ is of positive entropy as in 3.1. Assume further that $G \unlhd F \leq \operatorname{Aut}(X)$. Then we have:

(1) One has $\left|F: \operatorname{Stab}_{F}(M)\right| \leq 2$, so $\operatorname{Stab}_{F}(M)$ is normal in $F$. Write $\operatorname{Stab}_{F}(M)$ $=\left\langle h_{F}\right\rangle \ltimes T_{F}$ with $M=L\left(h_{F}\right)^{+}$, as in 3.1 .

(2) Suppose that $\left|F: \operatorname{Stab}_{F}(M)\right|=2$ and let $\tau \in F \backslash \operatorname{Stab}_{F}(M)$. Then $F=$ $\left\{\tau^{i} h_{F}^{j} t \mid i=0,1 ; j \in \mathbb{Z} ; t \in T_{F}\right\}$. Also $g \in F$ is of positive entropy if and only if $g=h_{F}^{j}$ t for some $j \neq 0$ and $t \in T_{F}$.

Proof. (1) We use Theorem 3.1 (and its notation) : $G=\left\langle h_{m}\right\rangle \ltimes T$. Write $h=h_{m}$ for simplicity. Take $f \in F$. Then $f h f^{-1}=h^{r} t$ for some $r=r(h) \in \mathbb{Z}$ and $t \in T$. Applying the equality to $M=L(h)^{+}$, we get $\left(f h f^{-1}\right)^{*} M \equiv \lambda^{r} M$ (with $\lambda=\lambda(h)>1$ ) and $h^{*}\left(f^{*} M\right) \equiv \lambda^{r}\left(f^{*} M\right)$. By the uniqueness Lemma 2.8, either $r=1$ and $f^{*} M$ equals $L(h)^{+}$, up to a positive scalar (so $f \in \operatorname{Stab}_{F}(M)$ ), or $r=-1$ and $f^{*} M$ equals $L(h)^{-}$, up to a positive scalar.

Suppose there are $f_{i} \in F$ such that $f_{i} h f_{i}^{-1}=h^{r_{i}} t_{i}$ as above, but with $r_{1}=r_{2}=$ -1. Then $\left(f_{2} f_{1}\right) h\left(f_{2} f_{1}\right)^{-1}=f_{2}\left(h^{-1} t_{1}\right) f_{2}^{-1}=\left(h^{-1} t_{1}\right)^{-1} t_{1}^{\prime}=h t^{\prime \prime} t^{\prime}=: h t^{\prime \prime \prime}$. Here $t_{1}^{\prime}=f_{2} t_{1} f_{2}^{-1}$ and $t^{\prime \prime \prime}$ are all in $T$. Hence, by the argument above, $f_{1} f_{2}$ (and especially $\left.f_{i}^{2}\right)$ are all in $\operatorname{Stab}_{F}(M)$. Thus (1) follows. Indeed, we may assume that $h_{m}=h_{F}^{\ell}$ for some $\ell \geq 1$, and $L\left(h_{F}\right)^{ \pm}=L(h)^{ \pm}$.

(2) The first part is from (1). Set $L^{ \pm}:=L\left(h_{F}\right)^{ \pm}$. We have $\tau T_{F} \tau^{-1}=T_{F}$ since $T_{F}$ is the set of all null entropy elements in $\operatorname{Stab}_{F}(M)$. Since $h_{F}$ is of positive entropy, 
by the proof of (1), we have $\tau h_{F} \tau^{-1}=h_{F}^{-1} t$ for some $t \in T_{F}$ and may assume that $\tau^{*} L^{+}=L^{-}$and $\left(\tau^{*} L^{-}=\right) \quad\left(\tau^{2}\right)^{*} L^{+} \equiv \alpha L^{+}$for some $\alpha>0$. We also have $\left(\tau^{2}\right)^{*} L^{-} \equiv \tau^{*}\left(\alpha L^{+}\right)=\alpha L^{-}$. By Lemma 2.12 and the uniqueness Lemma 2.8, we have $\alpha=1$, and hence $\tau^{2}$ (and also $\tau$ ) are of null entropy. Thus $\tau^{2}=t^{\prime} \in T_{F}$. Now for $t_{F} \in T_{F}$, we see that $\left(\tau h_{F}^{j} t_{F}\right)^{2}=\left(\tau h_{F}^{j} t_{F} \tau^{-1}\right) t^{\prime} h_{F}^{j} t_{F}=\left(h_{F}^{-1} t\right)^{j} t^{\prime \prime} h_{F}^{j} t_{F} \in T_{F}$ (here $\left.t^{\prime \prime}=\left(\tau t_{F} \tau^{-1}\right) t^{\prime} \in T_{F}\right)$, whence $\tau h_{F}^{j} t_{F}$ is of null entropy. (2) follows. The proposition is proved.

Let $\sigma: \operatorname{Aut}(X) \rightarrow \operatorname{Aut}(\mathrm{NS}(X))$ be the natural homomorphism. We have:

Proposition 3.4. Let $X$ be a smooth projective surface and $G \leq \operatorname{Aut}(X)$ a subgroup of positive entropy. Suppose that there is an $H \triangleleft G$ such that $G / H$ is abelian and $\sigma(H)$ is a finite subgroup of $\operatorname{Aut}(\operatorname{NS}(X))$. Then $G=\operatorname{Stab}_{G}(P)$ for every $P=L(g)^{ \pm}$ with $g \in G$ being positive entropy.

Proof. By Lemma 2.13, we have $h^{*} P \equiv P$ for every $h \in H$. By the assumption, for every $g_{1} \in G$ we have $g_{1} g=h g g_{1}$ for some $h \in H$. Applying this equality to $P=L(g)^{+}$, we get $g^{*}\left(g_{1}^{*} P\right) \equiv \lambda\left(g_{1}^{*} P\right)$ with $\lambda=\lambda(g)>1$. By the uniqueness Lemma 2.8, $g_{1}^{*} P$ equals $L(g)^{+}$(up to a positive scalar). So $g_{1} \in \operatorname{Stab}_{G}(P)$. Thus $G=\operatorname{Stab}_{G}(P)$. The case $P=L(g)^{-}$is similar. The proposition is proved.

In [24] $§ 7$, McMullen constructed rational surfaces $S^{v}$ for all $v \in\left(\mathbb{C}^{1, n}\right)^{*}$ there. The latter set contains all leading eigenvectors $v$ w.r.t. an eigenvalue $\beta$, for a Coxeter element $w$ in the Weyl group $W_{n}(n \geq 10)$ of the Minkowski parabolic lattice of rank $n+1$ (so $S^{v}$ has Picard number $n+1$ ). Here $v$ is a leading eigenvector for $w$ if $\beta$ is conjugate over $\mathbb{Q}$ to the largest (in terms of modulus) eigenvalue $\lambda(w)$ of $w$. One has $\lambda(w)>1$ (see [24] before 2.5).

\subsection{Proof of Theorem 1.5}

Set $S:=S^{v}$. Let $\sigma: \operatorname{Aut}(S) \rightarrow \operatorname{Aut}(\operatorname{Pic}(S))$ be the natural homomorphism. Since $S$ is rational, we do the identification: $\operatorname{NS}(X)=\operatorname{Pic}(S)=\mathbb{Z}^{1, n}$ and $(\operatorname{Pic}(S)) \otimes_{\mathbb{Z}} \mathbb{C}=$ $\mathbb{C}^{1, n}$, as in [24]. Set $k_{n}=\left[K_{S}\right] \in \operatorname{Pic}(S)$. By [24] Corollary 7.2, we have

$$
\operatorname{Aut}(S) \cong W_{n}^{v}=\left\{w^{\prime} \in W_{n} \mid[v] \in \mathbb{C}^{1, n} / \mathbb{C} k_{n} \text { is an eigenvector for } w^{\prime}\right\} .
$$

Since every $h \in \operatorname{Aut}(S)$ stabilizes $K_{S}$ and $K_{S}^{\perp}$, and $v \in K_{S}^{\perp}$, we have $\sigma(\operatorname{Aut}(S))$

$\subseteq \operatorname{Stab}_{\operatorname{Aut}(\operatorname{Pic}(S))}(v)$. So for every $h \in \operatorname{Aut}(S)$, we have $h^{*}(v)=\beta_{h} v$ for some $\beta_{h} \in \mathbb{C}$. Fix any $g \in \operatorname{Aut}(S)$ (of positive entropy) realizing the Coxeter element $w$. Set $\beta=\beta_{g}$. Let $f(x)=f_{g}(x)$ be the minimal polynomial of $\beta_{g}$ over $\mathbb{Q}$. Let $K\left(f_{g}\right)$, or $K\left(f_{g}, f_{h}\right)$ be the splitting field $(\subset \mathbb{C})$ of $f_{g}(x)$, or $f_{g}(x)$ and $f_{h}(x)$ over $\mathbb{Q}$. There is a $\tau=\tau_{g}$ in the Galois group $\operatorname{Gal}(f)=\operatorname{Gal}(K(f) / \mathbb{Q})$ of $f(x)$ such that $\tau(\beta)$ equals the spectral radius $\lambda=\lambda(g)=\lambda(w)$ (see Lemma 2.7). Clearly, we may take $v \in(\operatorname{Pic}(S)) \otimes_{\mathbb{Z}} K(f)$. Set $L^{ \pm}=L(g)^{ \pm} \in(\operatorname{Pic}(S)) \otimes_{\mathbb{Z}} \mathbb{Z}[\lambda]$ (see Proposition 2.11), so that $g^{*} L^{ \pm} \equiv \lambda^{ \pm} L^{ \pm}$.

We claim that $\operatorname{Aut}(S)=\operatorname{Stab}_{\operatorname{Aut}(S)}(M)$ for both $M=L^{ \pm}$, which will in turn imply the theorem by Theorem 3.1 and Proposition 2.11 .

Take $h \in \operatorname{Aut}(S)$. Extend $h^{*} \mid \operatorname{Pic}(S) \quad \mathbb{C}$-linearly to $h^{*} \mid(\operatorname{Pic}(S)) \otimes_{\mathbb{Z}} \mathbb{C}$. Denote by the same $\tau$ its extension to an automorphism of the splitting field $K\left(f_{g}, f_{h}\right)$ (by the isomorphism extension theorem). For a $\mathbb{C}$-divisor $D=\sum c_{i} D_{i}$ with $D_{i} \in \operatorname{Pic}(S)$ and 
$c_{i} \in K\left(f_{g}, f_{h}\right)$, define $\tau^{*}(D)=\sum \tau\left(c_{i}\right) D_{i}$. Then $\tau \circ h=h \circ \tau$. We have:

$$
\lambda \tau^{*}(v)=\tau^{*}(\beta v)=\tau^{*}\left(g^{*}(v)\right)=g^{*}\left(\tau^{*}(v)\right) .
$$

By Lemma 2.7, $\tau^{*}(v)$ equals the class of $\delta L^{+}$for some $\delta \in K(f)$ (by the choice of $v$ and $\left.L^{+}\right)$. Replacing $v$, we may assume that $\tau^{*}(v)$ equals the class of $L^{+}$. Now the claim for $M=L^{+}$(and hence the theorem) follows from the calculation below (noting that the case $M=L^{-}$is similar):

$$
h^{*} L^{+}=\tau^{*} h^{*}\left(\tau^{-1}\right)^{*} L^{+} \equiv \tau^{*} h^{*}(v)=\tau^{*}\left(\beta_{h} v\right)=\tau\left(\beta_{h}\right) \tau^{*}(v) \equiv \tau\left(\beta_{h}\right) L^{+} .
$$

This proves the theorem.

\section{Automorphisms of null entropy}

In this section, we consider groups of automorphisms of null entropy. The following is the main result of the section.

Let $\sigma: \operatorname{Aut}(X) \rightarrow \operatorname{Aut}(\operatorname{Pic}(X))$ be the natural homomorophism.

Theorem 4.1. Let $X$ be a smooth projective surface with irregularity $q(X)=0$ and let $G \leq \operatorname{Aut}(X)$ be a subgroup of null entropy such that $\sigma(G)$ is an infinte subgroup of $\operatorname{Aut}(\operatorname{Pic}(X))$. Suppose that the pair $(X, G)$ is minimal. Then we have:

(1) There is a nef $\mathbb{Q}$-divisor $M$ (which might be zero) with $M^{2}=0$, such that either $K_{X} \equiv M$ or $K_{X} \equiv-M$. In particular, $K_{X}^{2}=0$.

(2) If $X$ is rational, then $-K_{X}$ is nef and $X$ has an anti 1-canonical curve.

4.2. We need some preparation before starting the proof. For a smooth projective surface $X$, let $N_{1}(X)$ be the $\mathbb{R}$-vector space (of rank $\rho(X)$, the Picard number) of 1-cycles modulo numerical equivalence; let $\overline{\mathrm{NE}}(X)$ be the closure in $N_{1}(X)$ of the cone $\mathrm{NE}(X) \subset N_{1}(X)$ of effective 1-cycles (modulo numerical equivalence); see Kollar and Mori [21], Definitions 1.16 and 1.17. In our surface case, an $\mathbb{R}$-divisor $D$ is pseudoeffective if and only if $D \in \overline{\mathrm{NE}}(X)$.

Let $X$ be a smooth projective surface. Set $L=\mathrm{NS}(X) /$ tor, the Neron-Severi lattice modulo its (finite) torsion. Suppose that $G \leq \operatorname{Aut}(X)$ is of null entropy. Then the image $\bar{G}:=\operatorname{Im}(G \rightarrow \operatorname{Aut}(L))$ is of null entropy in the sense of Oguiso [27], 2 . Conversely, if $\bar{G}$ is of null entropy in the sense of Oguiso, then $G$ is of null entropy in the usual sense of Definition 2.5, by virtue of Lemma 2.8 .

We now assume that $q(X)=0$. Then $L=\operatorname{Pic}(X) /$ tor and $\operatorname{Pic}(X) \cong L \oplus$ (finite torsion subgroup). Clearly, $\sigma(G) \leq \operatorname{Aut}(\operatorname{Pic}(X))$ is infinite if and only if $\bar{G} \leq \operatorname{Aut}(L)$ is infinite.

For divisors $D_{1}, D_{2}$ we say $D_{1}=D_{2}$ in $L$ if their classes in $L$ are identical, i.e., if $D_{1} \equiv D_{2}$ (noting that $q(X)=0$ is assumed).

The following result is proved in Oguiso [27] Lemma 2.8. We slightly change the formulation, where $g(v)$ etc. should be understood as $\bar{g}(v)$ with $\bar{g}$ the image of $g$ via the surjective homomorphism $G \rightarrow \bar{G}$.

Lemma 4.3. The following are true.

(1) There is a unique ray $0 \neq \mathbb{R}_{>0} v \in \overline{\mathrm{NE}}(X)$ such that $v^{2}=0$ and $g(v)=v$ in $L$ for all $g \in G$. We can choose $v$ to be in $L$. 
(2) Suppose that $B$ is a subgroup of $O(L)$ and $\ell \in L$ such that $\ell^{2}>0$ and $b(\ell)=\ell$ for all $b \in B$. Then $B$ is finite.

We continue the proof of Theorem 4.1. Clearly, (2) is a consequence of (1) and the Riemann-Roch theorem. We now prove (1).

Let $V$ be in $\operatorname{Pic}(X)$ whose class in $L$ is $v$. We assert that $K_{X} \cdot V=0$. Suppose the contrary that $K_{X} . V \neq 0$. Then there is a positive integer $t$ such that $D=K_{X}+t V$ satisfies $D^{2}>0$. Clearly, $g(D)=D$ in $L$ for all $g \in G$. Then $\bar{G}$ is finite by Lemma 4.3 , contradicting the assumption on $\bar{G}$. So the assertion is true.

Next we assert that $V$ is nef. Let $V=P+N$ be the Zariski-decomposition. Since $g(v)=v$ in $L$ (and hence $g(V) \equiv V$ ) for every $g \in G$ and by the uniqueness in Lemma 2.3 , we have $g(N)=N$ and $g(P) \equiv P($ so $g(n P)=n P$ in $L$ for some positive integer $n$ with $n P$ integral) for every $g \in G$. If $P^{2}>0$, then we get a contradiction as above. So $P^{2}=0$. Now $0=v^{2}=(P+N)^{2}=N^{2}$ implies that $N=0$ and $V=P$ is nef. The assertion is proved.

Serre duality says $h^{2}\left(X, K_{X}+V\right)=h^{0}(X,-V)=0$ because $V . H>0$ for an ample divisor $H$ on $X$. Since $q(X)=0$, one has $\chi\left(\mathcal{O}_{X}\right)=1-q(X)+p_{g}(X)>0$. Now the Riemann-Roch theorem implies that $h^{0}\left(X, K_{X}+V\right) \geq \chi\left(\mathcal{O}_{X}\right)>0$. If $K_{X}+V \equiv 0$, then $-n K_{X} \sim n V$ for some integer $n>0$ because $q(X)=0$. We are done.

Suppose $K_{X}+V$ is not numerically trivial. Let $K_{X}+V=P^{\prime}+N^{\prime}$ be the Zariski decomposition. Since $g\left(K_{X}+V\right) \equiv K_{X}+V$ for all $g \in G$, we have, as above, $g\left(P^{\prime}\right)=P^{\prime}$ in $L, \quad\left(P^{\prime}\right)^{2}=0$ and $g\left(N^{\prime}\right)=N^{\prime}$, whence $G$ permutes components of $N^{\prime}$. By the uniqueness of $v$, we have $P^{\prime} \equiv a V$ for some $a \geq 0$. So $K_{X}+(1-a) V \equiv N^{\prime}$.

If $N^{\prime}=0$, then $-n^{\prime} K_{X} \sim n^{\prime}(1-a) V$ for some integer $n^{\prime}>0$ and we are done. Suppose that $N^{\prime} \neq 0$. Then $\left(N^{\prime}\right)^{2}<0$ and hence for some component $N_{1}$ of $N^{\prime}$, we have $0>N_{1} \cdot N^{\prime}=N_{1} \cdot\left(P^{\prime}+N^{\prime}\right)=N_{1} \cdot\left(K_{X}+V\right) \geq N_{1} \cdot K_{X}$. So $N_{1}$ is a $(-1)$-curve in $N^{\prime}$. Let $\Sigma$ be the set of $(-1)$-curves $N_{j}$ in $N^{\prime}$. Then $N_{i} \cap N_{j}=\emptyset(i \neq j)$ by the negativity of $N^{\prime}$. Since $G\left(N^{\prime}\right)=N^{\prime}$, we have $G(\Sigma)=\Sigma$, contradicting the minimality of $(X, G)$. The theorem is proved.

\section{Dynamics of groups; Proofs of some of Theorems $1.2-1.6$}

In this section we give dynamical structures for certain groups of surface automorphisms. We also prove Theorems 1.2, 1.3 and 1.6.

\subsection{Proof of Theorem 1.2.}

Clearly, there is a $G$-equivariant smooth blowdown such that the pair $(Y, G)$ is minimal. $G \leq \operatorname{Aut}(Y)$ is also of null entropy. So we may assume that $(X, G)$ is already minimal. Indeed, $\operatorname{Im}(G \rightarrow \operatorname{Aut}(\operatorname{Pic}(X)))$ is infinite if and only if so is $\operatorname{Im}(G \rightarrow$ Aut $(\operatorname{Pic}(Y)))$ since $G$ acts on the (finite) set of curves in the exceptional divisor $E$ of $X \rightarrow Y$ and $\operatorname{Pic}(X)$ is the direct sum of the pull back of $\operatorname{Pic}(Y)$ and the lattice generated by the curves in $E$. Set $\bar{G}=\operatorname{Im}(G \rightarrow \operatorname{Aut}(\operatorname{Pic}(X)))$. Consider the natural exact sequence:

$$
1 \rightarrow J \rightarrow G \rightarrow \bar{G} \rightarrow 1 .
$$

Assume that $\bar{G}$ is finite. Then $J$ is infinite because so is $G$. By Harbourne [16] Proposition 1.3, $X$ has only finitely many 'exceptional curves' (see [16] page 409, last paragraph for its definition) and our $J$ stabilizes each irreducible component of these 
exceptional curves. Inductively we can prove that there is a $J$-equivariant smooth blowdown $X \rightarrow Y$ onto a relatively minimal rational surface $Y$ (with $K_{Y}^{2}=8,9$ ). Now the assertion (1) follows from the Riemann-Roch theorem.

Assume $\bar{G}$ is infinite. Then Theorem 1.2 follows from Theorem 4.1.

Let $\sigma: \operatorname{Aut}(X) \rightarrow \operatorname{Aut}(\mathrm{NS}(X))$ be the natural homomorphism.

Proposition 5.2. Let $X$ be a smooth projective surface and $G \leq \operatorname{Aut}(X)$ a subgroup of positive entropy. Suppose that there is a sequence

$$
H \triangleleft A \unlhd G
$$

such that $\sigma(H)$ (resp. $\sigma(A))$ is a finite (resp. infinte) subgroup of $\operatorname{Aut}(\mathrm{NS}(X))$, and $A / H$ is abelian.

Then there is a $B \leq G$ fitting the sequence below and satisfying the four conditions below

$$
H \triangleleft A \unlhd B \unlhd G .
$$

(1) $|G / B| \leq 2$.

(2) $B=\left\langle h_{m}\right\rangle \ltimes T$, with $h_{m}$ positive entropy and $\sigma(T)$ a finite group.

(3) $A=\left\langle h_{m}^{a}\right\rangle \ltimes T_{1}$, with $a \geq 1$ and $T_{1} \unlhd T$.

(4) $H \unlhd T_{1}$ and $A / H=\left\langle\bar{h}_{m}^{a}\right\rangle \times(T / H)$, with $T / H$ abelian.

Proof. We claim that $A$ is of positive entropy. Suppose the contrary that $A$ is of null entropy. By Lemma 4.3 and the proof of Theorem $4.1(q(X)=0$ was used only in the calculation of the Riemann Roch theorem), there is a nef Cartier divisor $V$ such that $V^{2}=0$ and $a^{*} V \equiv V$ for all $a \in A$. Now for $g \in G$ and $a \in A$, write $g a g^{-1}=a^{\prime} \in A$. Then $\left(g a g^{-1}\right)^{*} V=\left(a^{\prime}\right)^{*} V \equiv V$ and $a^{*}\left(g^{*} V\right) \equiv g^{*} V$ for all $a \in A$. The uniqueness of the ray $\mathbb{R}_{>0}[V]$ in Lemma 4.3, implies $g^{*} V \equiv \alpha_{g} V$ for some $\alpha_{g}>0$. By the assumption on $G$, some $g \in G$ is of positive entropy, whence $L(g)^{ \pm}=V$, a Cartier divisor (Lemma 2.12). This contradicts Proposition 2.11. Thus the claim is true.

By Proposition 3.4, we have $A=\operatorname{Stab}_{A}(P)$ for every $P=L(g)^{ \pm}$with $g \in A$ being positive entropy. Set $B:=\operatorname{Stab}_{G}(P)$. By Proposition 3.3, we have $|G: B| \leq 2$ (hence $B \unlhd G$ ). Applying Theorem 3.1 to $B$ and $A$, we get (2) and (3). For (4), we have $H \leq T_{1}$ because $\sigma(H)$ is finite and hence $H$ is of null entropy. The proposition is proved.

As a consequence of Proposition 5.2, we have the result below:

Theorem 5.3. Let $X$ be a smooth projective surface and $G \leq \operatorname{Aut}(X)$ a subgroup of positive entropy. Suppose that there is an $H \triangleleft G$ such that $G / H=\mathbb{Z} \times \mathbb{Z}$ and $\operatorname{Im}(H \rightarrow \operatorname{Aut}(\mathrm{NS}(X)))$ is finite. Then we have:

(1) $G=\left\langle h_{m}\right\rangle \ltimes T$ and $H \unlhd T$.

(2) $\operatorname{Im}(T \rightarrow \operatorname{Aut}(\mathrm{NS}(X)))$ is finite.

(3) $G / H=\left\langle\bar{g}_{1}\right\rangle \times\left\langle\bar{g}_{2}\right\rangle$, with $g_{1}=h_{m}$ of positive entropy, and $g_{2} \in T$ of null entropy and infinte order.

For groups which are not necessarily of null entropy, we have Theorems 5.4 and 5.5 below, which are more general than Theorem 1.3 in the Introduction. 
A group $F$ is almost infinite cyclic if $F \cong \mathbb{Z} \ltimes F_{1}$ (semi-direct product) with $F_{1}$ finite (and normal in $F$ ).

Theorem 5.4. Let $X$ be a smooth projective surface and $G \leq \operatorname{Aut}(X)$ an infinite subgroup. Assume the following two conditions:

(1) There is an $H \unlhd G$ such that $G / H$ is soluble and the group $\operatorname{Im}(H \rightarrow$ $\operatorname{Aut}(\mathrm{NS}(X)))$ is finite; and

(2) No index $\leq 2$ subgroup of $G$ is almost infinite cyclic.

Then $G$ contains a subgroup $S$ of null entropy and infinite order.

In particular, when $X$ is rational, there is an $S$-equivariant smooth blowdown $X \rightarrow Y$ such that $Y$ has an anti-pluricanonical curve.

Proof. We may assume that $G$ is of positive entropy (see Theorem 1.2). Consider the derived series of $G$ :

$$
G=G^{(0)} \unrhd G^{(1)} \unrhd G^{(2)} \unrhd \ldots
$$

with $G^{(n)}=\left[G^{(n-1)}, G^{(n-1)}\right]$ the commutator subgroup of $G^{(n-1)}$. By the assumption, we have $G^{(n)} \leq H$ for some $n \geq 0$, whence $\sigma: \operatorname{Aut}(X) \rightarrow \operatorname{Aut}(\mathrm{NS}(X))$ maps $G^{(n)}$ to a finite group. Note that $\sigma(G)$ is infinte because $G$ is of positive entropy by the additional assumption. Thus there is a sequence below for some $r \geq 0$ :

$$
G^{(r+1)} \triangleleft G^{(r)} \unlhd G
$$

where $\sigma\left(G^{(r+1)}\right)$ is finite, $\sigma\left(G^{(r)}\right)$ is infinite, and $G^{(r)} / G^{(r+1)}$ is abelian.

Applying Proposition 5.2 to $H=G^{(r+1)}$ and $A=G^{(r)}$, we have $B=\operatorname{Stab}_{G}(P)=$ $\left\langle h_{m}\right\rangle \ltimes T$ and $|G / B| \leq 2$, as described there. If $T$ is infinite, then let it be $S$ and we are done. If $T$ is finite, then the index $\leq 2 \operatorname{subgroup} B$ of $G$ is almost infinite cyclic, contradicting the assumption of the theorem. This proves Theorem 5.4.

For a group $P$, we consider the upper central series:

$$
(1)=Z_{0}(P) \unlhd Z_{1}(P) \unlhd Z_{2}(P) \unlhd \cdots
$$

with $Z_{n}(P) / Z_{n-1}(P)$ equal to the centre $Z\left(P / Z_{n-1}(P)\right)$.

Theorem 5.5. Let $X$ be a smooth projective surface and $G \leq \operatorname{Aut}(X)$ an infinite subgroup. Assume the following two conditions:

(1) There is an $H \triangleleft G$ such that the group $\cup_{n=1}^{\infty} Z_{n}(G / H)$ is infinite and the group $\operatorname{Im}(H \rightarrow \operatorname{Aut}(\mathrm{NS}(X)))$ is finite; and

(2) No index $\leq 2$ subgroup of $G$ is almost infinite cyclic.

Then $G$ contains a subgroup $S$ of null entropy and infinite order.

In particular, when $X$ is rational, there is an $S$-equivariant smooth blowdown $X \rightarrow Y$ such that $Y$ has an anti-pluricanonical curve.

Proof. We may assume that $G$ is of positive entropy (see Theorem 1.2). Consider the upper central series of $\bar{G}:=G / H$ :

$$
(1)=Z_{0}(\bar{G}) \unlhd Z_{1}(\bar{G}) \unlhd Z_{2}(\bar{G}) \unlhd \ldots
$$

with $Z_{n}(\bar{G}) / Z_{n-1}(\bar{G})=Z\left(\bar{G} / Z_{n-1}(\bar{G})\right)$, the centre of $\bar{G} / Z_{n-1}(\bar{G})$. Write $Z_{n}(\bar{G})=$ $Z_{n} / H$ for some $Z_{n} \unlhd G$ with $Z_{0}=H$. By the assumption, $\left(\cup_{n \geq 1} Z_{n}\right) / H=\cup_{n \geq 1} Z_{n}(\bar{G})$ is infinite. So $\cup_{n \geq 1} Z_{n}$ is infinite. If $\sigma: \operatorname{Aut}(X) \rightarrow \operatorname{Aut}(\mathrm{NS}(X))$ maps every $Z_{n}(n \geq 0)$ 
to a finite group, then the group $\cup_{n \geq 1} Z_{n}$ has null entropy and let it be $S$, so we are done.

Therefore, we assume that there is a sequence below for some $r \geq 1$ :

$$
Z_{r-1} \triangleleft Z_{r} \unlhd G
$$

where $\sigma\left(Z_{r-1}\right)$ is finite, $\sigma\left(Z_{r}\right)$ is infinite, and $Z_{r} / Z_{r-1} \cong Z_{r}(\bar{G}) / Z_{r-1}(\bar{G})=$ $Z\left(\bar{G} / Z_{r-1}(\bar{G})\right)$ is abelian. The rest of the proof is identical to that of Theorem 5.4. This completes the proof of Theorem 5.5.

\subsection{Proof of Theorem 1.3.}

This theorem will follow from Theorem 5.4 or 5.5 , but we give a direct proof, so that we can see the dynamical structure of $G$. If $G$ is of null entropy, we let it be $S$ and the theorem is true. Assume that $G$ is of positive entropy. We apply Proposition 5.2 and use the notation there. Since $|G: A|=\infty$, we have $|B: A|=\infty$, so $\left|T: T_{1}\right|=\infty$. Thus $T$ is infinite and of null entropy; let it be $S$ and Theorem 1.3 is proved.

\subsection{Proof of Theorem 1.6.}

Set $H:=G \cap \operatorname{Ker}(\sigma)$. By the original Tits alternative theorem in [30] Theorem 1 , applied to $G / H \leq G L(\rho, \mathbb{C})$ with $\rho=\rho(X)$, either $G / H \geq \mathbb{Z} * \mathbb{Z}=\left\langle\bar{g}_{1}\right\rangle *\left\langle\bar{g}_{2}\right\rangle$, the non-abelian free group of rank 2 (so $G \geq \mathbb{Z} * \mathbb{Z}=\left\langle g_{1}\right\rangle *\left\langle g_{2}\right\rangle$ and $G$ satisfies the assertion (1) of the theorem), or $G / H$ is virtually soluble: there is a $G_{1} / H \leq G / H$ such that $\left|G: G_{1}\right|$ is finite and $G_{1} / H$ is soluble.

Let $\left\{g_{i} G_{1}\right\}$ be the (finite) set of all left cosets of $G_{1}$ in $G$. Define $G_{2}:=\cap g_{i} G_{1} g_{i}^{-1}$ $(\geq H)$. Clearly, $G_{2}$ is normal in $G$. Also $\left|G / G_{2}\right|$ is finite because all $\left|G: g_{i} G_{1} g_{i}^{-1}\right|$ are finite. This can be proved inductively by the injectivity of the following map between the sets of cosets: $\left\{g\left(J_{1} \cap J_{2}\right) \mid g \in G\right\} \rightarrow\left\{g J_{1} \mid g \in G\right\} \times\left\{g J_{2} \mid g \in G\right\}$, $x\left(J_{1} \cap J_{2}\right) \mapsto\left(x J_{1}, x J_{2}\right)$; here $J_{r} \leq G$.

Since $G_{1} / H$ is soluble so is its subgroup $G_{2} / H$. Since $G$ is of positive entropy, so is $G_{2}$ by the finiteness of $\left|G / G_{2}\right|$. By the proof of Theorem 5.4 applied to $G_{2}$ and $H$, there is a $B_{2}=\operatorname{Stab}_{G_{2}}(P)=\left\langle h_{2}\right\rangle \ltimes T_{2}$ such that $\left|G_{2} / B_{2}\right| \leq 2$.

Set $B=\operatorname{Stab}_{G}(P)$. If $B_{2}$ is normal in $G$, then the theorem (with the $B$ just defined) follows from Proposition 3.3 and Theorem 3.1.

Suppose $B_{2}$ is not normal in $G$. Then $\left|G_{2} / B_{2}\right|=2$ and let $\tau \in G_{2} \backslash B_{2}$. We apply Proposition 3.3 to $B_{2} \unlhd F:=G_{2} \leq \operatorname{Aut}(X)$ and use its proof. For any $g \in G$, $g h_{2} g^{-1}$ is in $G_{2}$ and of positive entropy, whence it equals $h_{2}^{r} t$ for some $r \in\{ \pm 1\}$ and $t \in T_{2}$. Also, if $r=1$, then $g \in B$; if $r=-1$ then $(\tau g) h_{2}(\tau g)^{-1}=\tau\left(h_{2}^{-1} t\right) \tau^{-1}=$ $h_{2} t_{1}\left(\tau t \tau^{-1}\right)=: h_{2} t_{2}$ with $t_{1}$ and $t_{2}$ in $T_{2}$, so that $\tau g \in B$; also $\tau^{2} \in T_{2} \leq B_{2} \leq B$. Thus $G / B=\langle\bar{\tau}\rangle \cong \mathbb{Z} /(2)$. Theorem 1.6 is proved (see Theorem 3.1).

\section{Positive entropy; proof of Theorem 1.7}

In this section, we consider surface automorphisms $g$ of positive entropy.

We prove Theorem 6.1 below, which will imply Theorem 1.7 in the Introduction. We also show that the two sets $\operatorname{Null}\left(L(g)^{ \pm}\right)$are identical (and finite) and equal to $\operatorname{Stab}(g)$; see Lemma 2.10.

Theorem 6.1. Let $X$ be a smooth projective rational surface with an automorphism $g$ of positive entropy. Assume the following two conditions: 
(1) The pair $(X,\langle g\rangle)$ is minimal; and

(2) Either the set $\operatorname{Stab}(g)$ of $g$-periodic curves contains a curve of arithmetic genus $\geq 1$, or $X$ has an anti-pluricanonical curve.

Then we have:

(1) There is a unique nonzero effective $\mathbb{Q}$-divisor $\Delta$ with $\operatorname{Supp}(\Delta) \subseteq$ $\operatorname{Stab}(g)$ such that $K_{X}+\Delta \equiv 0$. If $d$ is the smallest positive integer such that $d \Delta$ is a Cartier integral divisor, then $d\left(K_{X}+\Delta\right) \sim 0$. Further, the gcd of coefficients of $d \Delta$ is equal to 1.

(2) $\operatorname{Stab}(g)$ is a union of $\operatorname{Supp}(\Delta)$ and possibly a few $(-2)$-curves away from $\operatorname{Supp}(\Delta)$.

(3) One has $\kappa\left(X,-K_{X}\right)=0$, so every anti-pluricanonical curve is of the form $s(d \Delta)$ for some positive integer $s$.

(4) Suppose that $d \geq 2$. Then $\operatorname{Stab}(g)$ is a disjoint union of rational trees; we have $C^{2} \leq-2$ for all $C \in \operatorname{Stab}(g)$ and $C_{1}^{2} \leq-3$ for at least one $C_{1} \in \operatorname{Stab}(g)$.

We first prove the finiteness of the sets $\operatorname{Null}\left(L^{ \pm}\right)$; the finiteness of $\operatorname{Null}\left(L^{+}\right)$ $\cap \operatorname{Null}\left(L^{-}\right)$is known and easy; see section 2 for the notation.

Theorem 6.2. Let $X$ be a smooth projective surface with an automorphism $g$ of positive entropy. Set $L^{ \pm}=L(g)^{ \pm}$.

Then for each of $M=L^{+}$and $M=L^{-}$the set $\operatorname{Null}(M)$ is either empty or a finite set. In particular, $\operatorname{Null}\left(L^{+}\right)=\operatorname{Null}\left(L^{-}\right)=\operatorname{Stab}(g)$.

Proof. Clearly, there is a $\langle g\rangle$-equivariant smooth blowdown $\gamma: X \rightarrow Y$ (with $E_{\gamma}$ the exceptional divisor) such that $(Y,\langle g\rangle)$ is minimal. Also, $E_{\gamma} \subseteq \operatorname{Stab}(g)$. So $L^{ \pm} \cdot E_{1}=0$ for every $E_{1} \leq E_{\sigma}$ by Lemma 2.10. Thus $L^{ \pm}=\gamma^{*} L_{Y}^{ \pm}$for some nef divisor $L_{Y}^{ \pm}$on $Y$. The calculation (with $\lambda=\lambda(g)$ )

$$
\gamma^{*}\left(\lambda L_{Y}^{ \pm}\right)=\lambda L^{ \pm} \equiv g^{*} L^{ \pm}=g^{*} \gamma^{*} L_{Y}^{ \pm}=\gamma^{*}\left(g^{*} L_{Y}^{ \pm}\right)
$$

implies $g^{*} L_{Y}^{ \pm}=\lambda_{Y}^{ \pm}$because $\gamma^{*}:(\operatorname{Pic}(Y)) \otimes_{\mathbb{Z}} \mathbb{R} \rightarrow(\operatorname{Pic}(Y)) \otimes_{\mathbb{Z}} \mathbb{R}$ is injective. So $g$ is also of positive entropy on $Y$. By the projection formula, we have:

$$
\operatorname{Null}\left(L^{ \pm}\right)=\gamma^{-1}\left(\operatorname{Null}\left(L_{Y}^{ \pm}\right)\right) \cup \operatorname{Supp}\left(E_{\gamma}\right) .
$$

Thus we may assume that $(X,\langle g\rangle)$ is already minimal.

Assume the contrary that $\operatorname{Null}(M)$ contains infinitely many curves $C_{i}$. Since the Picard number $\rho(X)$ is finite, there are positive integers $r, s, a_{i}, b_{j}$ such that:

$$
D:=\sum_{i=1}^{r} a_{i} C_{i} \equiv \sum_{j=r+1}^{s} b_{j} C_{j} .
$$

This display shows that $D$ is nef and is not numerically trivial by the existence of an ample divisor $H$ on $X$. Also $M \cdot D=0$. So $D \equiv a M$ for some $a>0$ (Lemma 2.4). The Cartier-ness of $D$ contradicts Proposition 2.11 .

We study $\operatorname{Stab}(g)$ according to the arithmetic genera of its members.

Lemma 6.3. Let $X$ be a smooth projective surface with an automorphism $g$ of positive entropy. Let $C$ be a g-periodic curve. Then we have:

(1) The arithmetic genus $p_{a}(C) \leq 1$.

(2) If $p_{a}(C)=1$ then $X$ is rational. 
(3) Suppose that $(X,\langle g\rangle)$ is minimal and $p_{a}(C)=1$. Then $C$ is an anti 1canonical curve. $\operatorname{Stab}(g)$ is a union of $C$ and possibly a few $(-2)$-curves away from $C$.

Proof. By the assumption, we may fix some $s>0$ such that $g^{s}(C)=C$. If $X$ is irrational, then $C$ is a smooth rational curve; see e.g. Kawaguchi [18] Proposition 3.1. In particular, (2) is true.

We may assume that $X$ is rational. We follow Diller, Jackson and Sommese [10], Theorem 3.6. Considering the cohomology exact sequence associated to the exact sequence below:

$$
0 \rightarrow \mathcal{O}_{X}\left(K_{X}\right) \rightarrow \mathcal{O}_{X}\left(K_{X}+C\right) \rightarrow \mathcal{O}_{C}\left(K_{C}\right) \rightarrow 0,
$$

we get $p_{a}(C) \leq h^{0}\left(X, K_{X}+C\right)$ because $h^{1}\left(X, K_{X}\right)=q(X)=0$. If $\left|K_{X}+C\right|=\emptyset$ or $K_{X}+C \sim 0,(1)$ and (3) are true (see below).

Suppose that $0<D \in\left|K_{X}+C\right|$. Then $L . D=L . K_{X}+L . C=0$ by Lemma 2.9 and hence the intersection matrix of irreducible components of $D$ is negative definite by the Hodge index theorem or Lemma 2.10. Thus $1=h^{0}(X, D) \geq p_{a}(C)$ and also $g^{s}(D)=D$ as sets, since $\left(g^{s}\right)^{*} D \sim D$. This proves the assertion (1).

For (3), suppose $p_{a}(C)=1,(X,\langle g\rangle)$ is minimal and the above $D>0$. Note that $C$ is not a component of $D$, for otherwise $K_{X} \sim D-C \geq 0$, absurd (by (2)). Now $D^{2}<0$ and hence $0>D \cdot D_{1}=\left(K_{X}+C\right) \cdot D_{1} \geq K_{X} \cdot D_{1}$ for some component $D_{1}$ of $D$. Thus $D_{1}$ is a $(-1)$-curve. Note that this $D_{1} \in \operatorname{Stab}(g)$ because $g^{s}(D)=D$. This contradicts the minimality of $(X,\langle g\rangle)$ by Lemma 2.10 . Therefore, $K_{X}+C \sim 0$.

To prove the last part of $(3)$, let $C_{1}(\neq C)$ be in $\operatorname{Stab}(g)$. Then $C_{1}^{2}<0$ by Lemma 2.10 and $0=C_{1} \cdot\left(K_{X}+C\right) \geq C_{1} \cdot K_{X}$. Thus $C_{1}$ is a $(-2)$-curve by the minimality of $(X,\langle g\rangle)$. This proves the lemma.

Lemma 6.4. Let $X$ be a smooth projective surface with an automorphism $g$ of positive entropy. Suppose that $\operatorname{Stab}(g)$ is non-empty and consists of smooth rational curves. Then we have:

(1) Every curve $C$ in $\operatorname{Stab}(g)$ is a smooth rational curve with $C^{2}=-n$ for some $n \geq 1$.

(2) Suppose that $(X,\langle g\rangle)$ is minimal. Then in (1) we have $n \geq 2$ and $K_{X} \cdot C=$ $n-2 \geq 0$. There is a unique effective $\mathbb{Q}$-divisor $\Delta$ with $\operatorname{Supp}(\Delta) \subseteq \operatorname{Stab}(g)$ such that $\left(K_{X}+\Delta\right) \cdot C_{i}=0$ for every $C_{i}$ in $\operatorname{Stab}(g)$. The set $\operatorname{Stab}(g)$ is a union of $\operatorname{Supp}(\Delta)$ and possibly a few $(-2)$-curves away from $\operatorname{Supp}(\Delta)$.

Proof. (1) and the first part of (2) follow from Lemma 2.10 while the second of (2) is from solving linear equations and Zariski's lemma (Kollar and Mori [21] Lemma 3.41). Take $C$ in $\operatorname{Stab}(g) \backslash \operatorname{Supp}(\Delta)$. Then $C$ a $(-n)$-curve for some $n \geq 2$, and we have $0=C .\left(K_{X}+\Delta\right) \geq C . K_{X}=n-2 \geq 0$; so the last of (2) follows. The lemma is proved.

\subsection{Proof of Theorem 6.1.}

If $\operatorname{Stab}(g)$ contains a curve $C$ with $p_{a}(C) \geq 1$, then the theorem follows from Lemma 6.3.

So we may assume that $X$ has an anti-pluricanonical curve and $\operatorname{Stab}(g)$ (if not empty) consists of smooth rational curves $C_{i}$ (with $C_{i}^{2} \leq-2$ by Lemma 6.4). So 
$-d^{\prime} K_{X} \sim D^{\prime}$ for some $d^{\prime} \geq 1$ and effective (integral) Cartier divisor $D^{\prime}(\neq 0$, for $X$ being rational). By Lemmas $2.9-2.10$, we have $D^{\prime} \cdot L(g)^{ \pm}=-d^{\prime} K_{X} \cdot L(g)^{ \pm}=0$ and hence $\operatorname{Supp}\left(D^{\prime}\right) \subseteq \operatorname{Stab}(g)$. Since $K_{X}+\left(D^{\prime} / d^{\prime}\right) \sim_{\mathbb{Q}} 0$ and hence $\left(K_{X}+\left(D^{\prime} / d^{\prime}\right)\right) . C_{i}=0$ for every $C_{i} \in \operatorname{Stab}(g)$, we have $\Delta=D^{\prime} / d^{\prime}$ by the uniqueness in Lemma 6.4. Also (2) follows.

Write $\Delta=D / d$ with positive integer $d$ and effective Cartier integral divisor $D$ such that the gcd (called $r$ ) of coefficients of $D$ is coprime to $d$. We claim that $r=1$. Indeed, take a $(-1)$-curve $E$ (noting that $K_{X}^{2}<0$ by Lemma 2.9). We have $1=-K_{X} \cdot E=(D . E) / d$. Clearly, $r \mid D$.E. So $r \mid d$. Thus $r=1$.

Note that $d K_{X}+D=d\left(K_{X}+\Delta\right) \equiv 0$. So $n\left(d K_{X}+D\right) \sim 0$ for some positive integer $n$, since $q(X)=0$. Thus $d K_{X}+D \sim 0$ because $X$ is rational and hence $\operatorname{Pic}(X)$ is torsion free.

Since $\operatorname{Supp}(\Delta) \subseteq \operatorname{Stab}(g)$ is negative definite, we have $\kappa\left(X,-K_{X}\right)=0$. This proves (1). Now (3) follows.

For (4), we assert that $C_{i_{0}}^{2} \leq-3$ for at least one $i_{0}$. Otherwise, all $C_{i}$ are (-2)curves and hence $K_{X} \cdot \Delta=0$. This implies $0=\Delta \cdot\left(K_{X}+\Delta\right)=\Delta^{2}$, whence $\Delta=0$ by the negativity of $\operatorname{Supp}(\Delta) \subseteq \operatorname{Stab}(g)$. So $K_{X} \equiv 0$, absurd (for $X$ being rational). The assertion is proved.

Suppose that a reduced connected component $D$ of $\operatorname{Stab}(g)$ is either of non-simplenormal-crossing or contains a loop. Then $\left(K_{X}+D\right) . D \geq 0$ (i.e., $p_{a}(D) \geq 1$ ); see e.g. CCZ [8] Lemma 2.2. By the Riemann-Roch theorem, one has $K_{X}+D \sim G \geq 0$. Cancelling the common components of $D$ and $G$, one has $K_{X}+D^{\prime} \sim G^{\prime}$. If $G^{\prime}=0$ then one has a contradiction to that $d \geq 2$. Thus $G^{\prime} \neq 0$. By Lemma $2.9-2.10$, one has $L . G^{\prime}=L .\left(K_{X}+D^{\prime}\right)=0$ and that $\operatorname{Supp}\left(G^{\prime}\right) \subseteq \operatorname{Stab}(g)$ is negative definite. Hence $\left(G^{\prime}\right)^{2}<0$. So $0>G_{1} \cdot G^{\prime}=G_{1} \cdot\left(K_{X}+D^{\prime}\right) \geq G_{1} \cdot K_{X}$ for some component $G_{1}$ of $G^{\prime}$. Thus $G_{1}$ is a $(-1)$-curve in $\operatorname{Null}(L)$, contradicting the minimality of $(X,\langle g\rangle)$ (Lemma 2.10). So (4) is true. Theorem 6.1 is proved.

As an effective upper bound for the $d$ in Theorem 6.1, we have:

Remark 6.6. Let $X$ be a smooth projective rational surface with an automorphism $g$ of positive entropy. Assume the following three conditions:

(1) The pair $(X,\langle g\rangle)$ is minimal;

(2) Either the set $\operatorname{Stab}(g)$ of $g$-periodic curves contains a curve of arithmetic genus $\geq 1$, or $X$ has an anti-pluricanonical curve; and

(3) Either $\operatorname{Stab}(g)$ is contractible to quotient singularities, or $\operatorname{Stab}(g)$ is not a disjoint union of rational trees.

Then $X$ has an anti $s$-canonical curve for some $1 \leq s \leq 21$.

Indeed, As in Theorem 6.1, $d\left(K_{X}+\Delta\right) \sim 0$. We may assume that $d \geq 2$. Let $X \rightarrow \bar{X}$ be the contraction of $\Delta$ to quotient singularities. Then $\bar{X}$ is a rational log Enriques surface of index $d$ in the sense of [32] Definitions 1.1 and 1.4. Then by [32] and [3], we have $d \leq 21$.

Question 6.7. Does there exist an upper bound of $s$ as in Remark 6.6 without assuming the condition (3)? 


\section{References}

[1] W. Barth, K. Hulek, C. Peters and A. Van de Ven, Compact complex surfaces, Second edition, Springer-Verlag 2004.

[2] E. Bedford and K. Kim, Dynamics of Rational Surface Automorphisms: Linear Fractional Recurrences, math.DS/0611297.

[3] R. Blache, The structure of l.c. surfaces of Kodaira dimension zero. I., J. Algebraic Geom. 4 (1995), no. 1, 137-179.

[4] S. Cantat, Dynamique des automorphismes des surfaces projectives complexes, C. R. Acad. Sci. Paris Sr. I Math. 328 (1999), no. 10, 901-906.

[5] _ Dynamique des automorphismes des surfaces K3, Acta Math. 187 (2001), no. 1, 1-57.

[6] _ Sur la dynamique du groupe d'automorphismes des surfaces K3, Transform. Groups 6 (2001), no. 3, 201-214.

[7] S. Cantat and C. Favre, Symtries birationnelles des surfaces feuillete. J. Reine Angew. Math. 561 (2003), 199-235.

[8] J. A. Chen, M. Chen and D. -Q. Zhang, A non-vanishing theorem for Q-divisors on surfaces, J. Algebra 293 (2005), no. 2, 363-384.

[9] J. Diller and C. Favre, Dynamics of bimeromorphic maps of surfaces, Amer. J. Math. 123 (2001), no. 6, 1135-1169.

[10] J. Diller, D. Jackson and A. Sommese, Invariant curves for birational surface maps, Trans. Amer. Math. Soc. 359 (2007), no. 6, 2793-2991.

[11] T. C. Dinh and N. Sibony, Groupes commutatifs d'automorphismes d'une variété kählérienne compacte, Duke Math. J. 123 (2004), no. 2, 311-328.

[12] __ Une borne superieure pour l'entropie topologique d'une application rationnelle, Ann. of Math. 161 (2005), 1637-1644.

[13] S. Friedland, Entropy of algebraic maps, Proceedings of the Conference in Honor of Jean-Pierre Kahane (Orsay, 1993). J. Fourier Anal. Appl. 1995, Special Issue, 215-228.

[14] T. Fujita, On Zariski problem, Proc. Japan Acad. Ser. A Math. Sci. 55 (1979), no. 3, 106-110.

[15] M. Gromov, Entropy, homology and semialgebraic geometry, Sminaire Bourbaki, Vol. 1985/86. Astrisque 5 (1987), No. 145-146, 225-240.

[16] B. Harbourne, Rational surfaces with infinite automorphism group and no antipluricanonical curve, Proc. Amer. Math. Soc. 99 (1987), no. 3, 409-414.

[17] R. Hartshorne, Algebraic geometry, Graduate Texts in Mathematics, No. 52. Springer-Verlag, New York-Heidelberg, 1977.

[18] S. Kawaguchi, Projective surface automorphisms of positive topological entropy from an arithmetic viewpoint, math.AG/0510634.

[19] Y. Kawamata, K. Matsuda and K. Matsuki, Introduction to the minimal model problem, Algebraic geometry, Sendai, 1985, 283-360, Adv. Stud. Pure Math., 10, North-Holland, 1987.

[20] J. Keum, K. Oguiso and D. -Q. Zhang, Conjecture of Tits type for complex varieties and Theorem of Lie-Kolchin type for a cone, To Appear in Math. Res. Let., arXiv:math/0703103.

[21] J. Kollar and S. Mori, Birational geometry of algebraic varieties. Cambridge Tracts in Mathematics, 134, Cambridge University Press, Cambridge, 1998.

[22] B. Mazur, The topology of rational points, Experiment. Math. 1 (1992), no. 1, 35-45.

[23] C. McMullen, Dynamics on K3 surfaces: Salem numbers and Siegel disks, J. Reine Angew. Math. 545 (2002), 201-233.

[24] _ Dynamics on blowups of the projective plane, Preprint 2005.

[25] N. Nakayama and D. -Q. Zhang, Building blocks of étale endomorphisms of complex projective manifolds, RIMS preprint 1577 (yr 2007), Res. Inst. Math. Sci. Kyoto Univ. http://www.kurims.kyoto-u.ac.jp/preprint/index.html

[26] K. Oguiso, Tits alternative in hyperkähler manifolds, Math. Res. Lett. 13 (2006), 307 - 316.

[27] __ Automorphisms of hyperkähler manifolds in the view of topological entropy, Algebraic geometry, 173-185, Contemp. Math., 422, Amer. Math. Soc., Providence, RI, 2007.

[28] R. Salem, Algebraic numbers and Fourier analysis, Selected reprints, Wadsworth Math. Ser., Wadsworth, Belmont, CA, 1983.

[29] H. Sterk, Finiteness results for algebraic K3 surfaces, Math. Z, 189 (1985), no. 4, 507-513.

[30] J. Tits, Free subgroups in linear groups, J. Algebra, 20 (1972), 250 - 270. 
[31] Y. Yomdin, Volume growth and entropy, Israel J. Math. 57 (1987), no. 3, 285-300.

[32] D. -Q. Zhang, Logarithmic Enriques surfaces, J. Math. Kyoto Univ. 31 (1991), no. 2, 419-466.

[33] _ L Logarithmic Enriques surfaces. II, J. Math. Kyoto Univ. 33 (1993), no. 2, 357-397.

[34] _ Dynamics of automorphisms on projective complex manifolds, preprint 2006; also MPIM2007-19 at: www.mpim-bonn.mpg.de/Research/MPIM+Preprint+Series/

[35] _ Cohomologically hyperbolic endomorphisms of complex manifolds, MPIM2007-35.

Department of Mathematics

National University of Singapore, 2 Science Drive 2, Singapore 117543, Singapore AND

Universität Duisburg-Essen, Fachbereich Mathematik, 45117 Essen, Germany

E-mail address: matzdq@nus.edu.sg 